\title{
Complications and management of the neurologically impaired foot
}

M. R. C. MACDONALD*, J. W. BRANDSMA**,

A. G. WARREN***, H. CROSS+, R. J. SCHWARZ++,

S. SOLOMON+++, R. KAZEN\#, P. E. GRAVEM \& \&

H. SHRINIVASAN§

*The Leprosy Mission, Anandaban Leprosy Hospital, PO Box 151, Kathmandu, Nepal

**International Nepal Fellowship, Green Pastures Hospital and Rehabilitation Centre, PO Box 5, Pokhara, Nepal; Consultant to The Leprosy Mission International, Brentford, Middlesex, London, UK ***33 Centennial Ave, Chatswood 2067, Sydney, Australia

+Nepal Leprosy Trust, Lalgadh Hospital, Nepal

++International Nepal Fellowship, Green Pastures Hospital and Rehabilitation Centre, PO Box 5, Pokhara, Nepal

+++ SLRTC, Karigiri, Tamil Nadu, 632 106, India

\#ALERT, PO Box 165, Addis Ababa, Ethiopia

§Department of Plastic Surgery, Haukeland University Hospital, N-5021 Bergen, Norway

$\$ 11 B$ First Seaward Road, Chennai 600 041, India

\section{Ulcer aetiology and non-surgical management}

INTRODUCTION

There tends to be a commonly held belief in health education and Prevention of Impairment and Disability (POID) teaching, that health professionals, as the 'experts', need only to inform those with leprosy related impairments how to take care of themselves and the responsibility is passed on. It was recognized during the meeting that often, attitudes and behaviour of staff need to change when 'empowering' persons with impairments or activity limitations due to leprosy or nerve function impairments, for their own self-care. These changes include an understanding of adult learning and working alongside those people with impairments to bring about permanent changes in behaviour.

Correspondence to: M. R. C. Macdonald (e-mail: macdonald@ tlm.wlink.com.np) 
DISCUSSION

\section{Ulcer aetiology}

Risk factors in the aetiology of plantar ulceration include: ${ }^{9}$

- Loss of protective sensation

- Paralysis of intrinsic and extrinsic muscles (Srinivasan, 1964)

- Structural hyper/hypomobility

- Functional impairments

- Lifestyle

- Inadequate footwear

- Alteration of normal autonomic function resulting in anhidrotic skin. The consequent loss of elasticity compromises the skin, which may crack and fissure. Callosities become excessively dry and hard thereby creating a cause of localized high pressure

- Direct trauma or accidental trauma, e.g. treading on a tack, or impacting with excessive force

- Vascular and nutritional changes ${ }^{2}$

\section{Restricted hallux dorsiflexion}

Hallux limitus may be a risk factor in the aetiology of ulceration of the great toe. At least 60 degrees of hallux dorsiflexion is required for efficient propulsion. In order to affect adequate hallux dorsiflexion the 1st metatarsal must be stabilized in a plantar flexed position. If the 1st metatarsal is not adequately stabilized it may dorsiflex in response to ground reaction force during propulsion. The instability of the 1st metatarsal may result in an impingement of the proximal phalanx of the hallux against the head of the 1st metatarsal, thereby restricting hallux dorsiflexion. The demand for adequate dorsiflexion is met by forcing dorsiflexion at the IPJ of the hallux. A consequence is that a high focus of force is concentrated beneath the IPJ causing soft tissue breakdown. The effect of this problem is best reduced by addressing the causative factor rather than the effect. Hallux limitus is a common secondary effect of a foot that demonstrates aphasic subtalar pronation. This problem, when addressed by the timely use of an insole orthosis can be alleviated. An established osseous abnormality that has resulted from a hallux limitus is best addressed by the use of a curved 'roll over' sole. ${ }^{1}$ Alternatively, the impairment can be surgically addressed.

\section{Footwear}

Properly fitting footwear (shoes with a closed upper or sandals with a heel strap) is essential for the prevention of plantar ulceration. Where necessary, modifications can be made to address abnormalities. Modifications should be made inside the shoe at the foot/shoe interface and not outside the shoe except where a rocker sole is required. Moulded shoes have very little effect and are known to be stigmatizing. A simple MCR protective inlay may be as effective as a moulded shoe. ${ }^{7,8}$

In the first instance, modified footwear should be used. However there is a place to 'make the foot fit the shoe rather than the shoe fit the foot'. This may have benefit in areas of the world where:

a) only locally made shoes are available; 
b) wearing of special shoes is markedly stigmatizing or results in poor compliance;

c) adequate surgical facilities are available.

Corrective arthrodesis should be reserved for patients with severely deformed feet, or where ulceration has not been controlled with simple MCR and local shoes (with or without orthoses).

In patients with recurrent ulceration and failed conservative treatment, a corrective osteotomy or trimming of bony prominence can be performed. An additional soft tissue procedure, e.g. fascio cutaneous island flap, may be required. ${ }^{3}$

\section{Orthotic devices}

Subtle congenital and developmental impairments can place the insensate foot at further risk of plantar ulceration. It has been shown that certain orthotic devices designed to address these factors will facilitate healing and prevent re-ulceration. It is essential that where orthotic devices can be supplied, both the 'prescriber' and the appliance maker should understand the function of the 'ideal' foot because the objective of orthotic therapy is to assist the foot to function as close to the ideal as possible. Basic biomechanical examination and appliance manufacture training is essential if it is planned to establish a foot orthotic service. Patients need to realize that such orthotic appliances need to be worn at all times. If more than one pair of shoes is owned, appliances should be fitted to all shoes.

\section{Soaking}

To maintain the integrity of the skin of insensate feet, soaking and oiling is essential. The possible additional benefits of adding 'medication' to the water for soaking have not been shown. It was agreed at the meeting that nothing needs to be added to the water except the patient's feet, and that the oil, any oil, could be applied to wet feet. The soak rehydrates the dry skin and the oil retains the moisture. To oil without soaking is virtually a waste of oil! (NB some oil attracts rats).

\section{Dressings/medication}

In most instances, a clean dry dressing is sufficient to cover a plantar wound/ulcer. Saline may be used to clean wounds and in some instances (dry granulating wounds) saline dressings are indicated. Rest is essential. The rule should be not one step on an unprotected wounded sole. ${ }^{6}$

Generally speaking, no topical, systemic drugs or specialized dressing materials need to be used for wounds that are not infected. Only after radical ulcer debridement, when the integrity of unaffected tissue has been compromised, should an antiseptic such as betadine be used as prophylaxis (betadine is rapidly inactivated by body fluids).

Systemic antibiotics should be used only in cases of sepsis, lymphadenitis or lymphangitis. ${ }^{9}$ In diabetes, the use of antibiotics is more commonly needed. There is some evidence that the wide variety of interactive dressings available in the West may accelerate wound healing, but the cost of such is usually prohibitive for use in leprosy programs/hospitals. There is evidence that these dressings without adequate rest will not increase healing rates, even in sensate limbs. Most neuropathic ulcers will heal with rest, simple dressings and without antibiotics. Using unnecessary medication or specialized dressings also has the distinct 
disadvantage of creating dependency amongst those for whom self-care is the only sustainable long-term option. ${ }^{11}$

It should be noted that when surgery is indicated for drainage of pus or debridement of dead tissue, a period of time, usually 5-7 days, of immobilization with the limb in a splint and elevated in bed can localize the affected area and in many cases minimize the amount of debridement and hence tissue loss. If the pus collection is under pressure, then more urgent release may be indicated. Where possible, maintenance of anatomical structures should be pursued. It is preferable to return for a second debridement if needed rather than to take out tissues that do not really need to be removed. ${ }^{5}$

$P O I D$ - self care (in addition to the above)

In view of the integration of leprosy services into general health services, and the existence/ development of community based rehabilitation (CBR) programmes, the development of self-care/support groups should be encouraged. The functioning/dynamics of the groups should be the responsibility of the group itself.

The start of groups can be initiated from an existing POID program (officer) and together with the group members a 'curriculum' can be planned. The POID/officer/consultant will only have a facilitating role.

Where feasible and practical, self-care/support groups should consist of people with different impairments, activity limitations, and needs. ${ }^{4}$ People should be encouraged to do their own dressings at home to avoid hospitalization for minor lesions.

Training institutions and referral centres should be encouraged to develop self-care units. In these units, clients will be responsible for their own wound care (soaking/dressing), cooking, maintenance of quarters, gardening etc.

Do not leave POID self care until patients are ready for discharge. From day 1 of attending clinic or admission, start to teach the patient to 'soak scrape oil' (SSO) then dress and fully rest. Then (s)he will really leam how, and will be more likely to continue at home. ${ }^{10}$

\section{MANAGEMENT RECOMMENDATIONS}

Moulded insoles should only be used for a rigid deformed foot, and if used they should also be supplied with a rigid sole.

Ulcers should be cleaned with water or saline only. Where surrounding tissue is at risk, an antiseptic such as diluted betadine solution or a protective cream, such as zinc may be indicated. Antibiotics are only indicated where there is evidence of systemic infection, e.g. fever or lymphadenopathy, and are not a substitute for appropriate surgical debridement.

Self-care support groups can be very effective in the prevention and treatment of uncomplicated plantar ulcers in the community. These groups should be self-sustaining with input from outside facilitators only when required.

When practical/acceptable, total contact casts (TCC) or plaster of Paris moulded double rocker shoes (MDRS) should be applied to facilitate the healing of plantar ulcers. ${ }^{11,20,22}$

AREAS FOR RESEARCH

Further research is needed to determine the relative role of loss of protective sensation, 
intrinsic paralysis and structural and functional impairments in the aetiology of plantar ulceration.

A prospective trial of saline versus other additional wound solutions for soaking or dressing needs to be made. This should include local agents and solutions, easily available in local markets. ${ }^{9}$

Studies are needed to find out which patients benefit most (at what time) and for which reasons from self-care/self support groups. Which disciplines/professionals can be of help in changing attitudes and behaviour and how is this best done?

\section{Surgical management of motor loss}

INTRODUCTION

The correction of foot drop and claw toes by surgical means is well established. ${ }^{15,16}$ However, certain points of the procedures and their long-term results remain unknown.

\section{DISCUSSION}

\section{Surgical correction}

Tibialis posterior transfer (TPT) remains the operation of choice, for dynamic correction of foot drop in leprosy. ${ }^{12}$ There is no consensus regarding the route of the transferred tendon [inteiosseus (IO) versus circumtibial (CT)], or the site of insertion. Secondly, whether open or closed tendon Achilles lengthening (TAL) is required. There are 'indications' that the interosseus route may be preferred, but this has not been shown in a controlled study. However, it may result in a long-term better balanced foot with less inversion deformity. ${ }^{14}$

The important outcome measures are resting angle and active dorsi-flexion angle in swing through phase, and at heel strike. The aim of TPT is the restoration of normal gait. This is a more significant indicator than resting angles or range of motion, although these are all interrelated. Also important is the possible postoperative complication of inversion deformity.

Little is known about the effect of biomechanical changes, especially following changes to hind foot pronation following TPT.

\section{Surgery for the flail foot}

In exceptional cases when all the muscles below the knee are paralysed but the ankle passive range of motion is near normal, it is practical to tenodese the anterior tibial muscles and lengthen the Achilles tendon to provide a basically normal gait without evidence of foot drop.

\section{Footdrop spring}

A modified foot-drop strap is a good alternative for those who cannot/will not have foot drop surgery. This orthosis is worn on the distal lower leg only. The lining of the cuff should be of MCR and care should be taken that the distal rim of the cuff has many 2-cm deep incisions at 
1-cm intervals. This will prevent undue pressure on the dorsum of the foot. The modified orthosis can be worn under trousers. ${ }^{10}$

Compliance with this orthosis seems to be much higher than with the conventional below knee toe-raising spring ${ }^{18}$ or the above knee foot drop strap/spring which, when worn, is often not worn correctly (Lehman, personal communication).

Management of early foot drop by the use of steroids, a rest splint at night and a toeraising spring by day, can result in recovery in a large proportion of patients who when recently diagnosed may develop a permanent foot drop. All patients with a foot drop should be encouraged to wear a toe raising spring or other orthotic device, from diagnosis until surgery is available. ${ }^{17}$

\section{Claw toe deformity}

Mobile claw toes can be corrected by transfer of FDL to EDL (Girdlestone procedure). Fixed claw toes can be treated by resection of proximal phalanx and tenotomies, or PIPJ arthrodesis. In the presence of a reversed metatarsal arch, Srinivasan recommends proximal shift of EDL to metatarsal neck (sometimes combined with fusion of PIP joint) and shaving off the plantar side of the head of the metatarsals. This he sees as an alternative to the often-advocated metatarsal head resections. These procedures can be done at the time of the TPT operation or delayed if indicated.

\section{MANAGEMENT RECOMMENDATIONS}

Tendo-Achilles lengthening (open or closed) is recommended in all foot-drop corrections. During surgery aim to achieve 15-20 degrees of dorsi-flexion when the knee is out of its fixation splint. ${ }^{13}$

Tendon insertion should be in tendon(s) and/or Y ligament, not in the bone or periosteum. Resting angle following tendon suture should ideally be 15-20 degrees above right angle. Immobilization should be in maximum dorsi-flexion 15-20 degrees above right angle. A drop of 10-15 degrees within the first year post-surgery is common. ${ }^{13}$

It is important to analyse gait at postoperative follow-up and to see this as a key measure of successful outcome. A basic biomechanical examination is also necessary. The adjunctive use of in-shoe sole orthotic devices where indicated is also recommended.

AREAS FOR RESEARCH

It is recommended that a controlled study be conducted in which the two techniques of tibialis posterior tendon transfer (IO and CT) are compared with respect to outcome.

A comparative trial of short-term and long-term results of closed versus open-tendoAchilles lengthening is needed.

A standard gait and biomechanics assessment following tibialis posterior tendon transfer (TPT) needs to be developed for comparison of techniques used in surgery and comparing long-term outcomes.

To what extent is there phasic conversion of tibialis posterior muscle activity following transfer? Is TPT still useful as a static-supporting sling when phasic conversion is often not expected as in the elderly? 
What functional/structural consequences are there for the subtalar joint following tibialis posterior removal and should this be compensated for? Are additional orthoses required?

Does TPT change the frequency of metatarsal head and/or lateral boarder ulceration, or simply increase the number of heel ulcers?

\section{Neuropathic bone disintegration (NBD)}

\section{INTRODUCTION}

Neuropathic bone disintegration is a poorly recognized condition (especially in early presentation), with inadequately understood pathophysiology, often leading to delayed and incomplete treatment with serious long-term sequelae. The following discussion and recommendations attempt to clarify these areas and give clear guidelines for treatment.

The classic presentation of inflammation in any body part is heat, swelling, redness and pain. When in a neurologically impaired limb pain perception is compromised we must leam to assess the other features. ${ }^{23}$ If there are no other signs of infection, it is unlikely that painless local heat and swelling are due to infection. If in doubt, rest the limb and observe. With rest, any inflammation will localize and if heat and swelling (a hot spot/area) subsides in a few days and does not return when walking is resumed, it was unlikely to be a serious bone lesion or infection. Hence it was probably a relatively minor traumatic lesion, or minor infection usually requiring no further treatment. If doubts of severe infection such as osteomyelitis remain, treat as NBD but give antibiotics as well and keep in a total contact cast (TCC) for 6 weeks or longer and then review with new radiographs ( 2 and 6 weeks). An X-ray initially may not show any abnormal signs, but if there is NBD it may be recognized by about 6 weeks on X-ray due to the degree of osteopenia that has developed. X-ray signs of osteomyelitis may be evident from 2 weeks.

\section{DISCUSSION}

NBD should be suspected in every patient with a painless hot swollen foot or area of the foot that settles rapidly on full rest but returns as soon as walking resumes. The first sign of impending NBD is usually a 'hot spot/area' that is a localized area of heat usually with some swelling, when contrasted to the adjacent tissues or contralateral limbs, which indicates tissue pathology. Trained health workers and patients or their relatives can reliably assess these hot spots. These hot spots or local areas of inflammation occur from a variety of causes, but most commonly a bone lesion with active bone metabolism in response to some insult or injury. It is critical to detect these early, adequately immobilize and rest the foot, and allow sufficient time for bone healing and consolidation. If adequately treated, all will heal, but it is recognized that in a neurologically impaired foot (NIF) with NBD the bone healing may take 2-3 times longer for the bone lesion compared to normal. The sequence of treatment is three-fold: a period of

non-weight bearing or immobilization (to reduce swelling for cast application), followed by a period of protected weight bearing in a TCC and then trial walking.

If a patient presents with a warm swollen foot, routine examination ought to eliminate osteomyelitis. Elevated white cell count, positive microbiological cultures, lymphadenitis and lymphangitis on examination, and systemic symptoms are suggestive of osteomyelitis. 
Tagged white cell scans or bone scans are more conclusive. If these are positive, antibiotics should be started and the initial treatment includes rest with the foot splinted and elevated till the swelling decreases. If the swelling decreases within a few days and returns with walking, it is probably NBD, not osteomyelitis. Do not try to diagnose NBD by biopsy, as this may introduce infection and there is a great possibility that a biopsy will not remove a piece of definitely affected bone, which may be only a very small area. The biopsy will damage the bone and this encourages osteopenia around the biopsy area, which predisposes to NBD.

Bone can be involved in different ways in leprosy:

a) Direct infiltration by bacilli due to a deep septic ulcer may produce periostitis or osteomyelitis. Osteomyelitis results in osteopenia that predisposes to destruction and deformity.

b) Due to neurological deficit and repetitive stress that may lead to (stress or march) fractures.

c) Direct trauma leading to a fracture, (microscopic or macroscopic), especially when there is chronic hyperaemia, which can itself cause osteopenia predisposing to fractures.

d) Direct parasitization by leprosy bacilli causes cysts but rarely produces disintegration.

Predisposing factors for NBD are reduced pain perception in bones or joints and osteoporosis or osteopenia. Osteoporosis or osteopenia can occur due to prolonged immobilization in a cast ( 6 weeks in a cast can predispose to stress fractures on commencing unlimited walking), hyperaemia from any cause, e.g. infection, chronic lepra reaction, steroid therapy, age, calcium deficiency, diet and other causes.

It should be noted that although immobilization may further induce osteoporosis, immobilization is the treatment of choice in NBD. Immobilization is essential until disintegration has halted, and the broken bones have healed and are strong enough to stand the stress of usage. A radiographic examination cannot show definitely if the bone is fully healed. ${ }^{27}$ The most useful way of testing is by use the of the limb in trial walking.

\section{MANAGEMENT RECOMMENDATIONS}

When hot spots/areas are present, the foot should be treated as if NBD is present, until proven otherwise.

If a disintegrating bone lesion is suspected but cannot be confirmed by an X-ray and the foot is hot (swollen), it should be treated as if NBD is present. Rest and elevate, use a TCC and review in 6-8 weeks by radiograph and/or trial walking. ${ }^{19,22}$

If an X-ray can be made, it should be taken. Weight-bearing lateral (full length of foot) and AP-oblique X-rays will give the most information. ${ }^{28}$

The minimum length of time for immobilization in a total contact walking cast is 3 months for confirmed or minimal lesions. Advanced cases may require 9-12 months of immobilization. ${ }^{24,29}$

Recommended times of immobilization are:

a) Talo-calcaneal disintegration: 3-4 months.

b) Ankle disintegration: 6 months.

c) Mid-tarsal disintegration: 9-12 months.

d) Gross disorganization and or decalcification requires 12-15 months but should heal eventually.

With associated septic neuropathic disintegration, weight bearing is not allowed until the sepsis is controlled and all swelling subsided. 
Weight bearing in a TCC can be achieved by different means. A full length wooden foot piece or rocker is best to ensure the arch is supported as needed. ${ }^{20}$ An overshoe is useful in making walking easier, especially if both feet are affected. A rubber heel also plays a role. The use of a Bohler iron is advised, when there is destruction of the ankle or ulceration over the peak of a boat-shaped foot. Terrain and climate are variable, and probably will determine which device is best used for an individual to facilitate walking. ${ }^{27}$

Trial walking should be started after immobilization in a TCC. Trial walking starts for 3-5 min, with the use of suitable footwear, and then the patient rests for $2 \mathrm{~h}$. If there is no heat or swelling at the end of $2 \mathrm{~h}$, the patient can walk again for 3-5 min. Duration and frequency of trial walks can be slowly increased, until the patient can walk for $30 \mathrm{~min}$ (without heat and swelling of the foot), including stairs and rough terrain. These periods of walking can occur 3-4 times per day, once the patient learns to monitor themselves. If the foot becomes hot and swollen, even after reducing the duration of walking, it should be rested or again immobilized in a TCC. Trial walking could also be resumed when the foot is cool but with reduced frequency and duration. ${ }^{25,29}$ Recurrence of persistent heat and swelling suggests that there is still residual internal, pathology, not yet fully healed. Repeated recurrence of heat and swelling is best managed by a further 6-12 weeks in a TCC, followed again by trial walking. Patients with chronic cardiac or renal problems may well have repeatedly swollen ankles but there will be no heat if the swelling is unrelated to bone lesions.

A bi-valved modified total contact cast instead of a fixed TCC can sometimes be useful when expertise is available to make them, but not as a routine for active bone disorganization, disintegration or fractures. ${ }^{21,29}$

When end stage (consolidation, no further disintegration) is reached, the patient may require a moulded boot, and/or ankle brace or patellar weight-bearing device orthosis. ${ }^{21}$ However, in suitable patients in whom there has been adequate immobilization and trial walking has been completed successfully, no further device but a suitable shoe is required. If the disorganized foot can be moulded into a functional shape before the cast is applied, or can be surgically reconstructed, it should be possible for the patient to live the rest of his life in 'normal' shoes with a resilient insole. Following this, a carefully planned procedure with internal fixation of all osteotomies and prolonged immobilization can be made.

Corrective arthrodesis in a patient with NBD can be successful. It is indicated in a patient where without significant correction, the foot remains ulcer prone and nonfunctional. Surgery needs to be delayed at least 3 months, in a TCC from the time of presentation with acute disintegration, until initial heat and swelling has settled. This allows the active disintegration phase to settle and improves the likelihood of fusion. Following this, a carefully planned procedure with internal fixation of all osteotomies and prolonged immobilization, can be made. If the reconstruction is adequate and immobilization long enough the patient should not require special footwear afterwards. In some cultural groups, the use of prolonged casting is not acceptable and alternate methods of treatment may be used. ${ }^{26}$

\section{AREAS FOR RESEARCH}

- Does NBD occur in the presence of normal touch thresholds?

- To what extent is the lack of or diminished proprioception a risk factor in the development 
of a disintegrating bone/joint lesion? Does diminished/lack of propriocepsis and/or temperature sense occur independently from diminished/lack of protective sensation?

- Is there a delay in fusion if corrective arthrodesis is performed during healing in active NBD? Should surgery be delayed until complete healing has occurred? A study is needed, comparing the fusion rate, between corrective arthrodesis of deformed feet secondary to acute NBD performed at 3 months after the onset of treatment, versus the time for complete healing and consolidation without surgery.

- What footwear or orthotic devices are required for the ongoing management and protection of feet following healing after acute NBD? A comparison between the use of a fixed ankle brace verses footwear alone after treatment of NBD could also be performed.

\section{Surgical management of the anatomically deformed foot}

\section{INTRODUCTION}

Surgical procedures (soft tissue reconstruction, e.g. grafts and flaps, arthrodesis or amputations) may be required when the foot is structurally badly deformed. These procedures may also be indicated when there is little viable tissue for weight bearing, or the foot is very unstable.

\section{DISCUSSION}

\section{Soft tissue reconstruction}

The place of conservative treatment and/or a cast in the treatment of ulcers is well established. However, this often leads to healing with marked scarring. Pressure over bony prominences can lead to recurrent ulceration requiring hospital admission. A variety of soft tissue procedures from simple lateral calcaneal paring to more complex island vascular pedicle flaps can be used to improve the quality of soft tissue cover. Any soft tissue coverage needs to deal with the underlying bony prominences, usually with simple bone trimming. These flaps leave better quality weight bearing skin and in conjunction with good self care and appropriate footwear can prevent re-ulceration, prolonged foot survival and decrease in amputation rate. However, these do require expert surgical techniques and adequate facilities.

\section{Arthrodesis}

An arthrodesis or corrective osteotomy can be performed in the insensate foot, but immobilization needs to be $2-3$ times longer than in the normal sensate foot to achieve consolidation. $^{29,30}$

The aims of corrective arthrodesis are that the patient can become ulcer free, have a socially acceptable foot and walk in appropriate footwear, with, often, increased and appropriate weight-bearing surface. ${ }^{33}$

\section{Amputations}

Amputations are a last resort for the badly deformed and re-ulcerating foot. In confirmed 
cases of malignancy, amputation is usually indicated although there may be a place for local excision and soft tissue reconstruction.

The options for amputation include below knee (BK), tibio-calcaneal (T-C) (Pirigoff or Boyds) fusion, ${ }^{34}$ ankle dis-articulation (Symes), and a variety of forefoot amputations. Factors that should determine the level of amputation are the patients' preference, underlying pathology, and the skills of the prosthetist. A Symes amputation may require a patellar weight-bearing prosthesis. Re-ulceration is not uncommon. A T-C fusion does not usually require a prosthesis, because it provides a more stable heel pad. In a T-C fusion the leg is almost normal in length, initial immobilization in a walking plaster cast is required for the first 3 months, and then the patient is able to walk without additional support. Following a Symes amputation, they require additional support with crutches for the first 6 months. Both Symes and T-C fusion have a betterlong-term prognosis if the heel is still sensate. The ideal length for a BK stump for prosthetic fitting is $10-16 \mathrm{~cm}$. Some surgeons leave a longer stump and in all cases the prosthetist should be consulted on the ideall ength of stump for prosthesis. ${ }^{31}$

\section{MANAGEMENT RECOMMENDATIONS}

Recurrent/chronic foot ulcers can be managed with soft tissue reconstruction where indicated and surgical expertise is available. ${ }^{3}$

Corrective arthrodesis should be strongly considered as an alternative to radical amputation.

Often, racial/cultural variations in the acceptability of BK amputations (BKA) exist. It must be clearly seen that it is the patient's right to chose and the medical professional responsibility to enable that choice to be an informed decision, but seen as an option of last resort.

In many areas, the supply of orthotics and prosthetics is difficult, and repair and replacement in remote areas is impossible. The provision of a T-C fusion will allow the patient to use basketball boots that lace above the ankle so they do not need a prosthesis. Hence, it may well be the amputation of choice when the heel pad is good, and results have shown high patient acceptability. ${ }^{32}$

\section{AREAS FOR RESEARCH}

- A prospective comparative study of the long-term outcome of radical amputation procedures for squamous cell carcinoma of foot (BKA) versus local excision and reconstruction.

- A study looking at the long-term follow-up of a variety of soft tissue reconstruction and flaps for ulcers of the sole of the foot. This study would attempt to give guidelines for recommended treatment options; their indications and the skill base need for these procedures.

- Develop training programmes and ongoing supervision for surgeons involved in procedures requiring soft tissue reconstruction in feet.

\section{Acknowledgements}

The authors wish to thank The American Leprosy Mission for financial assistance in running 
the workshop, and Dr Han Tan for his invaluable assistance in writing the minutes of the workshop.

\section{References}

\section{Ulcer aetiology and non-surgical management}

${ }^{1}$ Birke JA, Patout CA, Foto JG. Factors associated with ulceration and amputation in the neuropathic foot. J Orthop Sports Phys Ther, 2000; 30: 91-97.

2 Bowering CK. Foot ulcers in patients with diabetes mellitus. Crit Rev Phys Rehab Med, 2000; 12: 25-49.

${ }_{3}$ Gravem PE. Heel ulcer in leprosy treated with fascio-cutaneous island flap from instep of the sole. Scand J Plast Reconstr Surg, 1991; 25: 155-160.

4 Kazen RO. Management of plantar ulcers in leprosy. Lepr Rev, 1999; 70: 63-69.

5 Kazen RO. Ulcer surgery for non-specialist surgeons. Lepr Rev, 1999; 70: 204-211.

${ }^{6}$ Mathew J, Antony P, Ethiraj T et al. Management of simple plantar ulcer by home based self-care. Ind J Lepr, 1999; 71: 173-187.

7 Seboka G, Saundersen P. Cost-effective footwear for leprosy control programmes: a study in rural Ethiopia. Lepr Rev, 1996; 67: 208-216.

8 Seboka G, Saundersen P, Currie H. Footwear for farmers affected by leprosy. Lepr Rev, 1998; 69: 182-183.

9 Srinivasan H. Do we need trials of agents alleged to improve healing of plantar ulcers? Lepr Rev, 1989; 60: 278282.

10 Srinivasan H. Prevention of disabilities in patients with leprosy. A practical guide. WHO, Geneva, 1993, p. 74.

11 Thomas S. Handbook of wound dressings. Macmillan, London, 1994.

\section{Surgical management of motor loss}

12 Malaviya GN. Surgery of foot drop in leprosy by tibialis posterior transfer. Lepr Ind, 1981; 53: 360-368.

13 Richard BM. Interosseous transfer of tibialis posterior for common peroneal nerve palsy. J Bone Joint Surg, 1989; 71B: 834-837.

${ }^{14}$ Soares D. Tibialis posterior transfer in the correction of foot-drop in leprosy. J Bone Joint Surg, 1996; 78B: 6162.

15 Srinivasan H, Mukherjee SM, Subramaniam RA. Two tailed transfer of tibialis posterior for correction of dropfoot in leprosy. J Bone Joint Surg, 1968; 50B: 623-628.

16 Warren AG. The correction of foot drop in leprosy. J Bone Joint Surg, 1968; 50B: 629-634.

17 Warren AG et al. A study of the incidence and outcome of foot weakness in leprosy. Lepr Rev, 1973; 44: $203-212$.

18 Warren AG. A foot drop spring. Lepr Rev, 1970; 41: 100-102.

\section{Neuropathic bone disintegration (NBD)}

19 Coleman WC, Brand PW, Birke JA. The total contact cast. J Am Pod Med Assoc, 1984; 74: 548-552.

20 Joseph B, Joshua S,Fritschi EP. The moulded double rocker plaster shoe in the field treatment of plantar ulcers. Lepr Rev, 1983; 54: 39-44.

21 Mehta JA, Brown C, Sargent N. Charcot restraint orthotic walker. Foot Ankle, 1998; 19: 619-623.

22 Mueller MJ, Diamond JE, Sinacore DR. Total contact casting in the treatment of plantar ulcers: controlled clinical trial. Diabetic Care, 1989; 12: 384-388.

23 Sinacore DR, Withrington NC. Recognition and management of acute neuropathic (Charcot) arthropathies of the foot and ankle. J Orthop Sports Phys Ther, 1999; 29: 737-747.

24 Warren AG. Tarsal bone disintegration in leprosy. J Bone Joint Surg, 1971; 53B: 688-695.

25 Warren AG. The management of tarsal bone disintegration Lepr Rev, 1972; 43: 137-147.

${ }^{26}$ Warren AG. The surgical conservation of the neuropathic foot. Ann R Coll Surg, 1989; 71: 236-242.

27 Warren AG. Plaster casts. Lepr Rev, 1971; 42: 55-59.

28 Warren AG, Haverson G. Tarsal bone disintegration in leprosy. Clin Radiol, 1979; 317-322.

29 Warren AG. The care of neuropathic limbs: a practical manual. Parthenon Publishing Group, New York, London, 1999. 
Surgical management of the anatomically deformed foot

${ }^{30}$ Ebenezer M, Partheebarajan S, Solomon S. Long-term follow up of joint stabilisation procedures in the treatment of fixed deformities of feet in leprosy. Lepr Rev, 1996; 67: 126-134.

31 Fleshman K, Fritschi E, Hill P. Rehabilitation by amputation. Lepr Rev, 1976; 47: 41-49.

32 Warren AG. The surgical conservation of the neuropathic foot. Ann R Coll Surg, 1989; 71: 236-242.

33 Warren AG. Surgery of leprosy. In: King M (ed) Primary surgery, vol 1, non-trauma. Oxford Medical Publications, 1990.

34 Warren AG. Conservative amputation of the neuropathic foot - the Pirigoff procedure. Oper Orthop Traum, 1997; 9: 49-58. 